\title{
Estudio comparativo entre técnicas quirúrgicas (incisión manual pequeña frente a facoemulsificación) para la cirugía de catarata
}

\section{Comparative study between surgical techniques (manual small incision versus phacoemulsification) for cataract surgery}

\author{
Carla R. Robles-Gutiérrez ${ }^{1 *}$, Mariana Moctezuma-Dávila², Horacio A. Meza-Velarde ${ }^{1 \dagger}$, \\ Araceli Martínez-Castrellón ${ }^{1}$, Martha G. Rangel-Charqueño ${ }^{1}$, Aldanely Padrón-Salas ${ }^{1}$ y \\ Gustavo S. Moctezuma-Bravo ${ }^{3}$
}

${ }^{1}$ Hospital Central Dr. Ignacio Morones Prieto, Universidad Autónoma San Luis Potosí; ${ }^{2}$ Facultad de Medicina, Universidad Autónoma San Luis Potosi; ${ }^{3}$ Hospital General Zona No.50, IMSS. San Luis Potosí, México. ${ }^{\dagger}$ Fallecido el 29 de Septiembre de 2017

\begin{abstract}
Resumen
La cirugía de catarata se practica mundialmente. Objetivo de estudio: Determinar los resultados posquirúrgicos de agudeza visual, pérdida de células endoteliales y opacidad de la cápsula posterior, entre dos técnicas quirúrgicas que corrigen la catarata. Tipo de estudio: Ensayo clínico controlado, aleatorizado y doble ciego, en pacientes con diagnóstico de catarata, con seguimiento a 18 meses. Criterios de exclusión: Pacientes con retinopatía diabética proliferativa, edema macular, ambliopía y glaucoma. Criterios de eliminación: Pacientes que requirieron cambio de técnica quirúrgica y pacientes que no acudieron a su seguimiento. Tamaño de la muestra: 75 pacientes según estudios de Peduzzi y Frank. Consideraciones bioéticas: estudio aprobado por el Comité de Investigación y Bioética Institucional Hospitalaria, siguiendo lineamientos de Helsinki, código de Núremberg y Ley General de Salud, donde cada paciente firmó su consentimiento informado. Análisis de datos: Datos descritos con medidas de tendencia central, dispersión y proporciones. Análisis estadístico: empleó Chi cuadrada, prueba exacta de Fisher, $T$ de Student, Anova y regresión lineal. Resultados: Se incluyeron finalmente 57 pacientes, 35 mujeres y 22 hombres, 26 diabéticos y 8 con retinopatía diabética no proliferativa. Tratados 34 con incisión manual pequeña (IMP) y 23 con facoemulsificación (FACO), su edad promedio grupal fue de 70.4 y 67.9 años, respectivamente. Las variables agudeza visual y opacidad de la cápsula posterior resultaron estadísticamente significativas para ambas técnicas. El resultado fue: disminución del conteo de células endoteliales para las dos técnicas, mayor cambio del eje queratométrico en la IMP y mayor polimorfismo de endotelio y paquimetría corneal en la FACO. Conclusión: La IMP no es inferior a la FACO.
\end{abstract}

Palabras clave: Cirugía. Catarata. Facoemulsificación. Incisión manual pequeña. Ensayo clínico controlado.

\begin{abstract}
Cataract surgery is practiced worldwide. Objective: To determine the postoperative results of visual acuity, endothelial cell loss and posterior capsule opacity between two cataract surgery techniques. Type of Study: Randomized, controlled, dou-
\end{abstract}

Correspondencia:

${ }^{*}$ Carla R. Robles-Gutiérrez

C. Barrio de Tlaxcala 116

Col. San Pedro Fecha de recepción: 27-12-2017

Disponible en internet: 05-09-2018

San Luis Potosí, México

Fecha de aceptación: 05-07-2018

Rev Mex Oftalmol. 2018;92(5):239-246

E-mail: carla_rrg@ @otmail.com

DOI: $10.24875 /$ RMO.M18000043

www.rmo.com.mx

0187-4519/○ 2018 Sociedad Mexicana de Oftalmología. Publicado por Permanyer México. Este es un artículo Open Access bajo la licencia CC BY-NC-ND (http://creativecommons.org/licenses/by-nc-nd/4.0/). 
ble-blind clinical trial (ECCA), in patients with cataract, with an 18-month follow-up. Exclusion Criteria: Patients with proliferative diabetic retinopathy, macular edema, amblyopia and glaucoma. Elimination Criteria: Patients that required a change in the surgical technique or that did not complete follow up. Sample Size: 75 patients according to Peduzzi and Frank studies. Bioethical Considerations: Study approved by the Research and Bioethics Institutional Committee. Following the Helsinki, Code of Nuremberg and General Law of Health guidelines, all the patients signed an informed consent form. Data are described with measures of central tendency, dispersion and proportions. Statistical analysis used Chi-square, Fisher's exact test, Student's $t$, ANOVA and linear regression tests. Results: 57 patients were included: 35 women and 22 men, 26 diabetics and 8 with non-proliferative diabetic retinopathy. 34 patients were treated with manual small incision (IMP) and 23 with phacoemulsification (FACO). The average age group was 70.4 and 67.9 years, respectively. Visual acuity and posterior capsule opacification were statistically significant for both techniques. A decrease in endothelial cell count was observed for the two techniques, but a greater change in the keratometry axis was present in IMP. In addition, endothelial polymorphism and corneal pachymetry were increased in FACO. Conclusion: IMP is not inferior to FACO.

Key words: Surgery. Cataract. Phacoemulsification. Manual small incision. Controlled clinical trial.

\section{Introducción}

La catarata u opacidad del cristalino es la principal causa de ceguera reversible $e^{1,2}$. En el mundo hay aproximadamente 37 millones de personas con ceguera ${ }^{1}, y$ se calculan 515 mil personas con ceguera en México, donde el $60 \%$ son debidas a catarata, agregándose de 50 a 60 mil nuevos casos por año ${ }^{3}$.

En oftalmología, la extracción quirúrgica de catarata es la cirugía más practicada a nivel mundial, y puede ser mediante incisión manual pequeña (IMP), extrayendo el núcleo a través de un túnel esclerocorneal de $6.5 \mathrm{~mm}$; por facoemulsificación (FACO), a través de una incisión de $3 \mathrm{~mm}$ y usando ultrasonido que emulsifica la catarata; y la extracción extracapsular de catarata (EECC), a través de una incisión de $12 \mathrm{~mm}$. La más frecuentemente empleada es la FACO, le sigue la EECC y al final la IMP. Esta última se realiza con mayor frecuencia en países en desarrollo por sus ventajas de ser más económica, ya que no se utiliza ultrasonido, se puede emplear lente rígido 0 uno plegable ${ }^{4,5}$ y porque no es inferior a la $\mathrm{FACO}^{6,7,8}$. Sin embargo, las tres técnicas mejoran la calidad de vida del paciente al permitir la recuperación de la visión. En la IMP y la $\mathrm{FACO}^{9}$ no se emplea sutura y requieren menor tiempo de rehabilitación visual. El tiempo promedio de cirugía en la IMP es de 8 minutos y 35 segundos, mientras que en la FACO es de 15 minutos y 30 segundos $^{4}$. Sin embargo, la curva de aprendizaje para la realización de la FACO requiere de mayor tiempo ${ }^{2,4,9}$.El presente estudio, mediante un ensayo clínico y empleando las técnicas de IMP y FACO para la corrección quirúrgica de catarata con seguimiento de diez y ocho meses, busca comparar los resultados de agudeza visual mejor corregida (AVMC), diferencia de la pérdida de células endoteliales y opacidad de la cápsula posterior (OCP).

\section{Objetivo}

Determinar si la IMP tiene resultados posquirúrgicos similares a la FACO.

\section{Material y métodos}

\section{Diseño del estudio}

Ensayo clínico controlado aleatorizado (ECCA), doble ciego, realizado del 1 de mayo de 2013 al 31 de marzo de 2015, en el Hospital Central Dr. Ignacio Morones Prieto en San Luis Potosí, México.

\section{Criterios de inclusión}

Pacientes con diagnóstico de catarata candidatos a manejo quirúrgico, con agudeza visual $(\mathrm{AV})<20 / 40$.

\section{Criterios de exclusión}

Pacientes con retinopatía diabética proliferativa avanzada, edema macular, degeneración macular relacionada con la edad, ambliopía, glaucoma, pacientes que no tienen conteo de células endoteliales o este es mayor a 3 meses previos a la cirugía y aquellos que no acepten participar en el estudio.

\section{Criterios de eliminación}

Pacientes que requieran cambio de técnica quirúrgica, patología macular al extraer la catarata y pacientes que no acudan a sus consultas de seguimiento.

\section{Tamaño de la muestra}

Considerando la AV final corregida (técnica quirúrgica), diferencia en la disminución de células endoteliales 
(técnica quirúrgica) y opacidad de la cápsula posterior (técnica quirúrgica), para estudios que emplean modelos de regresión según Peduzzi, Feinstein y Frank ${ }^{10,11}$ son entre 40 y 80 repeticiones. Variables: sexo, edad, catarata $u$ opacidad del cristalino medida en la escala internacional estandarizada: Lens Opacities Classification System (LOCS); que evalúa en seis imágenes de lámpara de hendidora, el color de núcleo (NC1-6), de la opalescencia (NO1-6), y de cinco imágenes, la catarata cortical (C1-5) y la catarata subcapsular (P1-5) III (NC1-6, NO1-6, C1- 5 y P1-5); AV: capacidad visual medida con y sin corrección (logMAR); conteo de células endoteliales: células endoteliales obtenidas por técnica manual mediante microscopia especular cel/ $/ \mathrm{mm}^{2}$; OCP: grado de opacidad de la cápsula posterior a 3 y 18 meses después de la cirugía, según la escala de LOCSIII P1-5; queratometrías $(\mathrm{Ktm})$ : promedio de dioptrías corneales en sus $3 \mathrm{~mm}$ centrales; refracción o equivalente esférico (EE): AV con corrección logrando su máxima capacidad visual representada por su EE: E + 1/2 C; presión intraocular (PIO) partiendo de $1 \mathrm{mmHg}$, normal 10-21.

\section{Consideraciones bioéticas}

Estudio aprobado por el Comité de Investigación y Bioética del Hospital Central Dr. Ignacio Morones Prieto, número de registro 55-13. Siguiendo lineamientos de Helsinki, código de Núremberg y Ley General de Salud. Cada paciente firmó su consentimiento informado, donde la información obtenida se manejó de manera confidencial y se mantuvo su identidad de forma anónima.

\section{Recursos materiales}

Facoemulsificador Stellaris-pc, B\&L. US- modo A, Alcon; microscopio especular, Konan; microscopio oftalmológico, Leica; proyector de agudeza visual, Huvitz; tonómetro de Goldman, Keeler; lente PMMA, Acuity; lente plegable hidrofílico, B\&L; instrumental oftalmológico del Hospital Central Dr. Ignacio Morones Prieto; y computadora laptop, HP.

\section{Técnica de muestreo}

Con lista de números aleatorios para asignar pacientes a cirugía A o B. Estandarización de la intervención: se realizó una exploración oftalmológica completa inicial, tomando en cuenta antecedentes de diabetes mellitus y presencia de retinopatía diabética proliferativa o no proliferativa. Previo a la cirugía, se tomó la agudeza visual sin corrección (AVSC) y estenopeico a $3 \mathrm{~m}$ con proyector calibrado en logMAR, refracción y $\mathrm{Ktm}$ en autorrefracto-queratómetro calibrado tomando el EE, el promedio de las Ktm y la PIO con tonómetro de Goldman calibrado, y microscopia especular con técnica manual (un día previo a la cirugía). Se llevó el procedimiento quirúrgico como cirugía ambulatoria. Todas las operaciones con la técnica de incisión manual pequeña $(\mathrm{IMP})^{2}$ fueron realizadas por un solo cirujano, Dr. Meza $\left(3^{\star}\right)$, las de facoemulsificación (FACO) ${ }^{12}$ también fueron realizadas por otro único cirujano, Dra. Martínez agudeza mejor corregida (AMC) $\left(4^{*}\right)$. En el postoperatorio inicial, los pacientes se trataron con antibiótico y antiinflamatorios esteroideos tópicos. Su control se evaluó a los siete y treinta días de la cirugía con registro de AV y PIO. Al tercer mes se registró AVMC, PIO, Ktm, OCP (según LOCS III) y microscopia especular. Estas pruebas se realizaron con microscopio especular, Konan, proyector de agudeza visual CCP-3100, Hivitz, autorefracto-queratómetro KR-3000, Topcon y tonómetro de aplanación de Goldman, Keeler. A los 18 meses de seguimiento se estudio: AVSC/AVMC y OCP.

\section{Análisis de los datos}

Las variables cuantitativas se describieron mediante medidas de tendencia central y dispersión; las variables cualitativas mediante proporciones; el análisis estadístico con paquete STATA v.12 ${ }^{\circledR}$, considerando significancia estadística $p<0.05$, y empleando Chi cuadrada, prueba exacta de Fisher, t de Student, Anova y regresión lineal.

\section{Resultados}

Inicialmente se incluyeron 75 pacientes. Se excluyeron 18 pacientes: Nueve porque no se les realizó la cirugía el día marcado por alteraciones sistémicas, falta de tiempo quirúrgico y falta de insumos para la cirugía. Cinco, por no cumplir su seguimiento. Dos, al convertir su cirugía en extracción extracapsular, uno por hipema que ameritó lavado de cámara anterior y otro por edema macular no valorado prequirúrgicamente por opacidad del cristalino. Finalmente 57 ojos de diferentes pacientes se incluyeron en el estudio, a 34 de ellos se les realizo la técnica de IMP y a 23 la de FACO.

Los pacientes de la técnica de IMP tuvieron una edad promedio de 70.4 años, con desviación estándar (DE) de 12.3, 16 pacientes padecían diabetes y, de estos, cinco con retinopatía diabética no proliferativa (RPDNP), 22 del género femenino y fueron 17 ojos derechos y 17 izquierdos. 
Los pacientes de la técnica de FACO tuvieron una edad promedio de 67.9 años, DE de 14.2, diez con diabetes

Tabla 1. Datos generales

\begin{tabular}{|l|c|c|}
\hline & $\begin{array}{c}\text { Pacientes y } \\
\text { IMP }\end{array}$ & $\begin{array}{c}\text { Técnica 0x } \\
\text { FAC0 }\end{array}$ \\
\hline Sexo & 34 & 23 \\
\hline Femenino & $22(64.7 \%)$ & $13(56,5 \%)$ \\
\hline Masculino & $12(35.3 \%)$ & $10(43.5 \%)$ \\
\hline Ojos & 34 & 23 \\
\hline Derechos & $17(50 \%)$ & $13(56,5 \%)$ \\
\hline Izquierdos & $17(50 \%)$ & $10(43.5 \%)$ \\
\hline Diabéticos & 21 & 13 \\
\hline Ds/RPNNP & $16(47 \%)$ & $10(43 \%)$ \\
\hline Dc/RPDNP & $5(14.7 \%)$ & $3(13 \%)$ \\
\hline
\end{tabular}

Dc/RPDNP: diabéticos con retinopatía diabética no proliferativa; Ds/RPDNP: diabéticos sin retinopatía diabética no proliferativa;

FACO: facoemulsificación; IMP: incisión manual pequeña; Qx: quirúrgica. mellitus y tres de ellos con RPDNP, 13 del género femenino y fueron 13 ojos derechos y 10 izquierdos (Tabla 1). Con los análisis estadísticos empleados, las variables estadísticamente no significativas en la técnica de IMP entre el promedio inicial y final fueron: EE: +0.10 y -0.99 , respectivamente, Ktm: 43.83 y 43.84, y presión Intraocular: 13.91 y 12.36. En la técnica con FACO fueron: EE de -0.07 y -0.60 , Ktm: 43.53 y 43.37 y PIO: 12.43 y 11.86 , como promedios inicial y final, respectivamente. La variable AV inicial contra siete, treinta, noventa y quinientos cuarenta días posquirúrgicos resultaron estadísticamente significativos tanto para la técnica de IMP como para la técnica de FACO. Otras variables que también tuvieron diferencia estadística significativa, tanto con la técnica de IMP como con la técnica de FACO, fueron el conteo de células endoteliales, eje queratometrico y OCP (Tabla 2). Resaltamos: La disminución del conteo de células endoteliales por ambas técnicas, el mayor cambio del eje queratométrico en IMP y el mayor polimorfismo de endotelio y paquimetría corneal en FACO.

Tabla 2. Resultados promedio por tipo de técnica quirúrgica

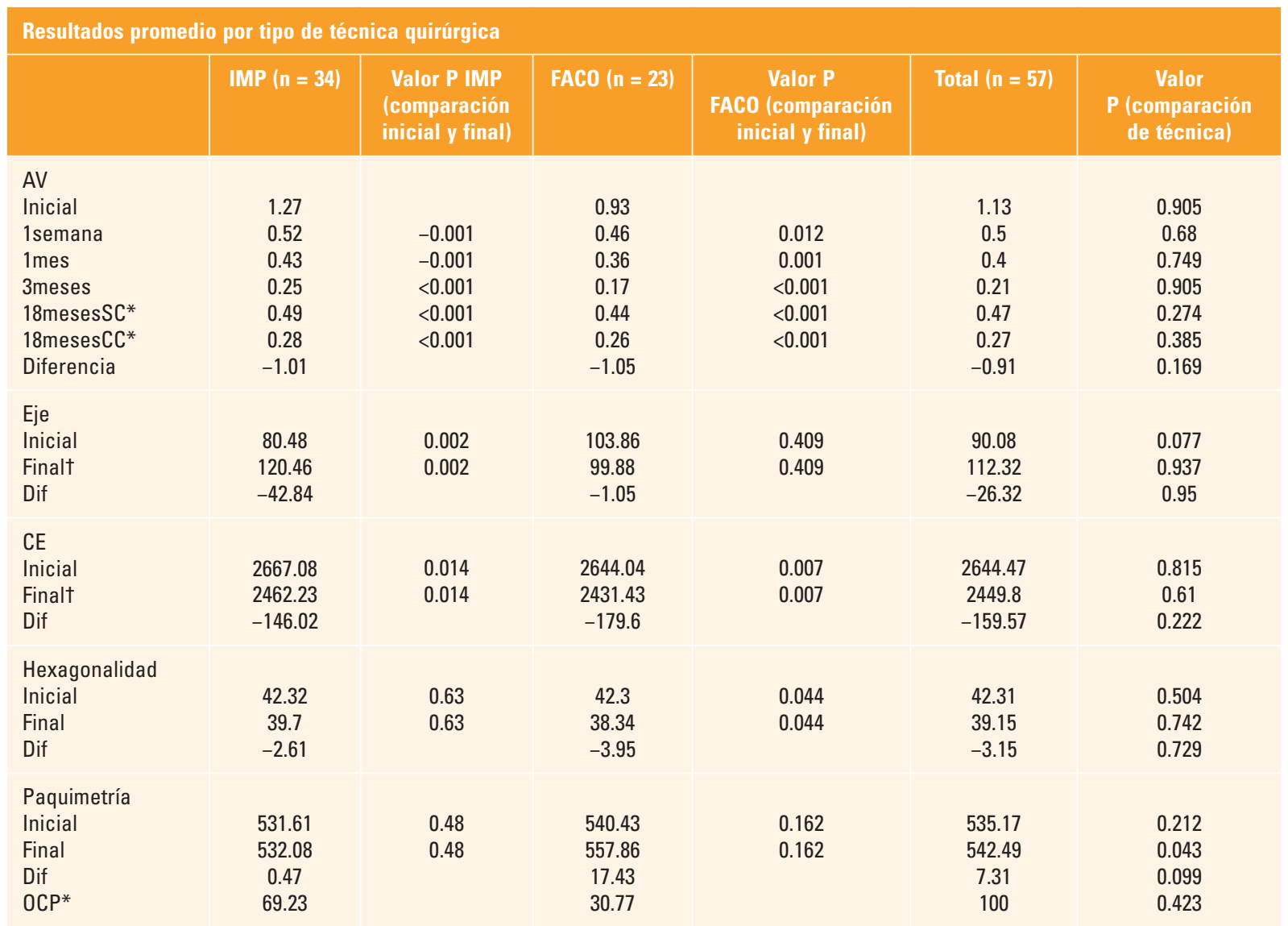

*Seguimiento a 18 meses. IMP $n=26$, FACO $n=17$.

${ }^{\dagger}$ Seguimiento a seis meses.

AV: agudeza visual; CE: células endoteliales; Dif: diferencia; EE: equivalente esférico; FACO: facoemulsificación; IMP: incisión manual pequeña; Ktm: keratometrías; OCP: opacidad de la cápsula posterior. 
Tabla 3. Análisis de la dureza del cristalino por técnica quirúrgica

\begin{tabular}{|l|c|c|c|c|c|}
\hline Dureza & MP & FAC0 & Total & AV Log Mar* & Prom Fin AV p* \\
\hline Blanda & $18(49 \%)$ & $19(51 \%)$ & $37(65 \%)$ & 0.21 y 0.30 & 0.021 \\
\hline Dura & $16(80 \%)$ & $4(20 \%)$ & $20(35 \%)$ & 0.18 y 0.13 & 0.021 \\
\hline
\end{tabular}

Prom Fin AV p*: promedio final de agudeza visual con valor de p; Blanda: LOCS III (N01-N03); Dura: LOCS III (N03.1-N06)

AV: agudeza visual; FACO: facoemulsificación; IMP: incisión manual pequeña.

Respecto a la dureza blanda (NO1-NO3) y dura (NO3.1NO6) del cristalino, según LOCS III, fueron 18 cataratas blandas operadas por IMP y 19 por FACO; mientras que de las cataratas duras, 16 se operaron por IMP y 4 por FACO. De los resultados obtenidos no se observó una relación de la dureza del cristalino con la AV final en cada una de las técnicas (Tabla 3). En las variables AV previa a la cirugía, pacientes sin diabetes mellitus y pacientes con diabetes mellitus con retinopatía diabética leve sin afección macular, se encontró la peor AV al inicio del estudio en diabéticos sin retinopatía diabética, con un promedio de visión de 1.61 logMAR, seguido por los no diabéticos, con un promedio de 0.93 logMAR, y, finalmente, los diabéticos con retinopatía diabética leve sin afección macular, con un promedio 0.84 logMAR, observando una diferencia estadísticamente significativa por obtener una $p=0.0298$. Variables: agudeza visual final y variable agudeza visual previa a la cirugía presentó una $r 2<0.001$ $(p=0.968)$, por tanto no existe relación.

El análisis estadístico de las variables que fueron estadísticamente significativas se muestran en las figuras 1, 2, 3 y 4 .

\section{Discusión}

La AV posterior a una cirugía de catarata es el resultado de varios factores, como el poder queratométrico, ejes corneales, calidad de la película lagrimal y longitud axial del globo ocular, que a su vez influyen en el tipo de lente intraocular a emplear.

En nuestro estudio la variable AV final en el seguimiento posquirúrgico a 3 meses fue similar en IMP y FACO, y se lograron promedios de AVMC de 0.21 logMAR para ambos grupos. Existen reportes con resultados similares $2,6,7,8,13$. Sin embargo, grupos de FACO mantuvieron una AV sin mejor corrección que los grupos de IMP, pero sin tener diferencias estadísticamente significativas, como tampoco lo tuvieron en los resultados visuales entre pacientes diabéticos y no diabéticos, por lo que pueden intervenirse a pacientes que no tengan datos de actividad de retinopatía diabética.

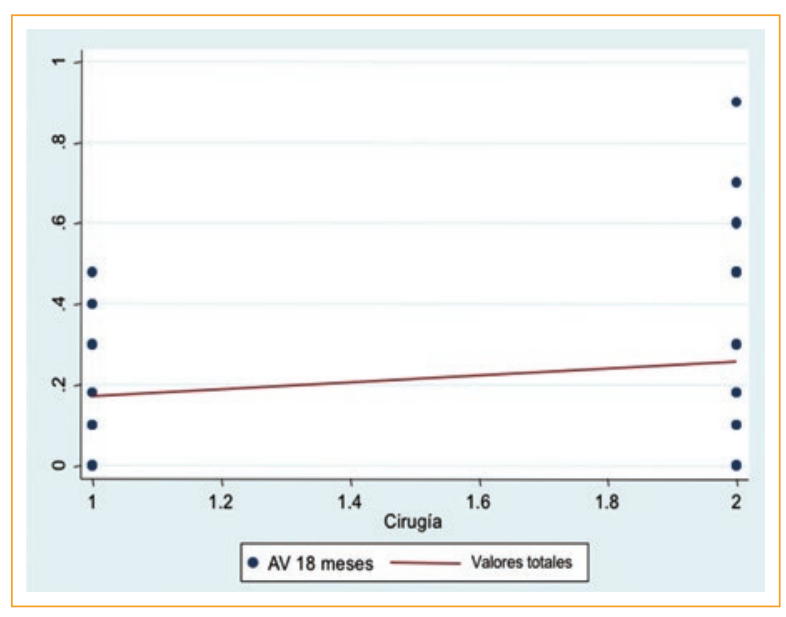

Figura 1. Análisis de regresión lineal entre variables: AV final y tipo de técnica quirúrgica efectuada. Se obtuvo una $r^{2}$ de $0.046(p=0.108)$, lo que indica que no existe relación entre ellas. AV: agudeza visual.

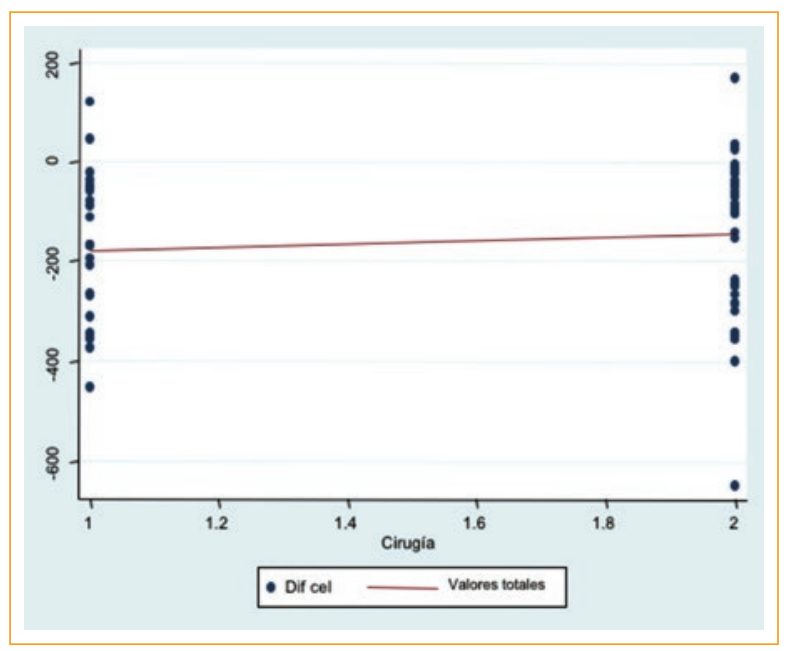

Figura 2. Análisis de regresión lineal entre diferencia de pérdida de células endoteliales y tipo de técnica quirúrgica. Se encontró una $r^{2}$ de $0.010(p=0.445)$, por lo que tampoco tienen relación.

El estudio ${ }^{14}$ comparó IMP y FACO en relación con AV final y no encontró diferencias estadísticamente significativas entre ambos grupos. Los resultados de 


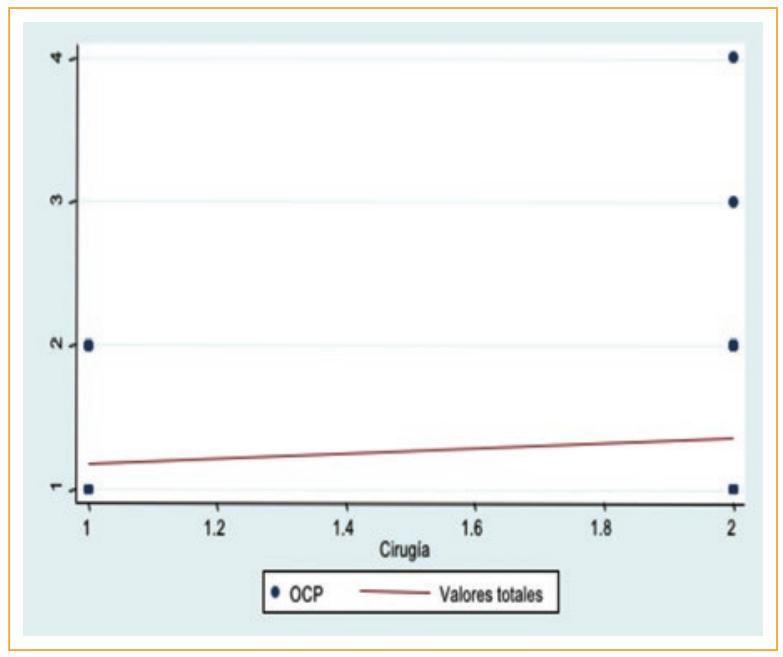

Figura 3. Análisis de regresión lineal entre opacidad de la cápsula posterior y la técnica quirúrgica. No se encontró relación al obtener una $r^{2}$ de 0.022 ( $\left.p=0.265\right)$. Estos resultados fueron similares a los tres meses y a los diez y ocho meses de seguimiento. OCP: opacidad de la cápsula posterior.

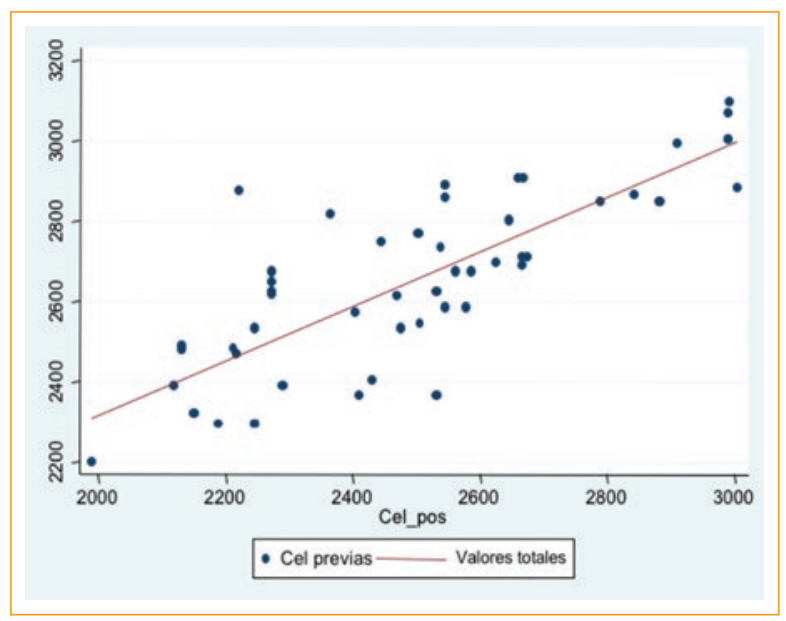

Figura 4. Análisis de regresión lineal entre número de células endoteliales posteriores a la intervención y el número de células endoteliales previas a la cirugía. Se obtuvo una $r^{2}$ de $0.645(p<0.001)$, lo que significa que el número de células posteriores a la intervención depende del número de células previas a la cirugía, pero no se relaciona con el tipo de cirugía realizada.

AV a seis semanas posteriores a la cirugía de catarata, con FACO como estándar de oro contra IMP, mediante ECCA, encontraron visión mejor o igual a 20/60 en el grupo FACO, con una diferencia estadísticamente significativa ${ }^{9}$.

La cirugía de catarata es uno de los factores que causan la pérdida de células endoteliales y el mejor indicador de estrés endotelial es el polimorfismo, dado por la hexagonalidad (\% de hexagonalidad normal > $60 \%$ ) y por la variación en el tamaño celular del coeficiente de variación (CV) (cifra CV normal <33\%) 6,14,15,16. Después de la cirugía de catarata, las células endoteliales presentes se elongan y desplazan para mantener la continuidad endotelial, generando cambios en la densidad celular, morfología hexagonal y variación del tamaño celular.

Este trauma quirúrgico conlleva una pérdida celular con disminución de su morfología hexagonal y aumento en la variación del tamaño de estas células en un intento de sustituir a las células perdidas, condicionando un aumento del CV y una disminución de la hexagonalidad, que se manifiesta con un aumento del polimegatismo y polimorfismo. En nuestro estudio se encontró mayor polimorfismo en FACO que en IMP, el cual fue estadísticamente significativo $(p=0.044)$; $\sin$ embargo, para el polimegatismo no. Esta pérdida celular puede llevar a edema corneal prolongado, que en casos graves ocasiona descompensación corneal asociada a baja visual ${ }^{6,13,17,18}$.Un estudio $\mathrm{ECCA}^{6}$ en 200 pacientes comparó el porcentaje de pérdida de células endoteliales con FACO vs. IMP y encontró pérdida de células endoteliales sin diferencia estadísticamente significativa entre ambas cirugías. Pero sí existió esta en cada una de las técnicas quirúrgicas (IMP y FACO), lo que demuestra que efectivamente después del trauma quirúrgico existe una disminución estadísticamente significativa del número de células, tal como encontramos en nuestro estudio (IMP, $p=0.014$; FACO, $p=0.007$ ). Otro estudio comparó el porcentaje de pérdida de células endoteliales posterior a EECC, IMP y FACO, pérdidas con disminución del 4.72, 4.21 y $5.41 \%$, respectivamente, que no tuvieron diferencias estadísticamente significativas.

En estudios a 3 meses posquirúrgicos en poblaciones sin antecedente de patología ocular, la cirugía de catarata disminuyó la densidad de células endoteliales, aumentó su coeficiente de variación y disminuyó el porcentaje de células hexagonales ${ }^{13,19,20}$. Se ha comparado el porcentaje de pérdida de células endoteliales entre FACO e IMP a 6 semanas posquirúrgicas, obteniendo una pérdida del 15.5 y del $15.3 \%$, respectivamente, sin tener diferencia estadística ${ }^{6,18}$.

En nuestro estudio existió una pérdida de células endoteliales a 3 meses de seguimiento en promedio de 146 células (7.5\%) para IMP y de 179 células (6.8\%) para FACO, lo cual tampoco tuvo una diferencia estadísticamente significativa. 
Un estudio a tres meses posquirúrgicos en pacientes diabéticos y no diabéticos sometidos a IMP y FACO encontró un mayor índice de pérdida de células endoteliales en el grupo de pacientes diabéticos: $6.2 \%$ vs. $1.4 \%$ en pacientes no diabéticos, según aumento del grado de la catarata y duración de la cirugía; sin embargo, esta diferencia no fue estadísticamente significativa ${ }^{13}$.

La OCP es una complicación frecuente posterior a la cirugía de catarata, con una incidencia entre el 2 y el 15\% a un año de la cirugía con facoemulsificación y entre el 2 y $63 \%$ a 3 años de la cirugía. Un estudio que investigó la incidencia y factores de riesgo para OCP ${ }^{21}$ con seguimiento a 3 años concluyó que el cirujano en entrenamiento, el uso de lentes hidrofílicos de una y tres piezas y de lentes hidrofóbicos de bordes romos, comparados con lentes de acrílico hidrofóbicos de bordes en escuadra, son factores predisponentes de OCP. Ruit ${ }^{22}$ comparó la aparición de OCP en IMP y FACO en 6 meses de seguimiento posquirúrgico, encontró $26.1 \%$ de OCP en IMP y $14.6 \%$ en FACO, con un mayor grado de opacidad en IMP.

En nuestro estudio, el grado de opacidad de la cápsula posterior no se presentó más en un grupo que en otro. Sin embargo, se pudo observar que el mayor grado de opacidad se presentó en el grupo de IMP, aunque tendría que realizarse un mayor análisis tomando en cuenta otros factores ya conocidos como predisponentes de OCP, como el tipo y diseño de lente intraocular, edad, sexo, entre otros ${ }^{21}$. Pero para fines de nuestro estudio, solo se relacionó la OCP con la técnica quirúrgica utilizada (IMP o FACO).

Se midió el EE antes y después de la cirugía, al igual que las Ktm y su eje. El promedio del EE fue de 0.60 D y 0.99 D, para IMP y FACO, respectivamente, sin tener diferencia estadísticamente significativa. Las Ktm para ambos grupos prácticamente se mantuvieron iguales como antes de la cirugía. Respecto al eje, sí existió cambio de 42.84 grados respecto al eje previo a la cirugía en IMP, con estadística significativa $(p=0.002)$. Lo que podría justificar la mejoría de la AV en el grupo de la FACO sin corrección, no estadísticamente significativa.

Otro hallazgo importante fue en la paquimetría. Se publicó un estudio en el 2005 en el que los pacientes con un conteo endotelial previo a la cirugía de $640 \mu \mathrm{m}$, distrofia endotelial de Fuchs y la presencia de algún síndrome iridocorneoendotelial se encontraban con un mayor riesgo de descompensación corneal posterior a un trauma quirúrgico, por lo que se recomendó realizar procedimientos triples para estos pacientes ${ }^{6,13,23}$.En nuestro estudio se comparó la paquimetría previa a la cirugía y a los 3 meses; sin embargo, no hubo una diferencias estadísticamente significativas de la paquimetría inicial y final entre ambos grupos, aunque encontramos que los pacientes intervenidos por FACO tuvieron un aumento estadísticamente significativo $(p=0.043)$ en la paquimetría en comparación a la técnica de IMP.

Se esperaba encontrar que quizás las cataratas con mayor dureza pudieran tener mejor resultado si fuesen intervenidas mediante IMP y lo contrario para FACO, es decir, que las cataratas blandas tuvieran un mejor resultado con FACO, sin embargo, esto no fue así. Y la PIO no presentó variaciones estadísticamente significativas posteriores a la cirugía en relación con la tomada al inicio del estudio para ambos grupos.

\section{Conclusión}

Si bien nuestro estudio, aunque con un número pequeño de pacientes, mostró resultados similares a lo publicado. La cirugía de catarata por IMP no es inferior a la FACO en cuanto a la AV, pérdida de células endoteliales ni opacidad de la cápsula posterior, y sus diferencias presentes no fueron estadísticamente significativas.

Aunque la AVMC al cabo de 3 meses fue muy parecida en ambos grupos, obteniendo un EE similar. En el eje queratométrico del grupo de IMP tuvo diferencias estadísticamente significativas.

La OCP fue similar en ambos grupos, sin embargo, el mayor grado de se presentó en el grupo de IMP, pero sin que la diferencia fuera estadísticamente significativa.

Los resultados de la cirugía en los pacientes diabéticos y no diabéticos no tuvieron diferencia con una técnica $u$ otra al ser intervenidos, como tampoco existieron variaciones de la PIO en ambos grupos.

Por lo tanto, se reconoce la ventaja de la técnica de IMP al obtener resultados visuales aceptables, en caso de no contar con una máquina de facoemulsificación o cualquiera de sus insumos requeridos para efectuar la técnica de FACO. Siendo fundamental una adecuada selección del caso, efectuar una cirugía cuidadosa y llevar un seguimiento posterior a la cirugía para obtener un buen resultado, sea cual fuere la técnica empleada.

\section{Responsabilidades éticas}

Protección de personas y animales. Los autores declaran que los procedimientos seguidos se 
conformaron a las normas éticas del comité de experimentación humana responsable y de acuerdo con la Asociación Médica Mundial y la Declaración de Helsinki.

Confidencialidad de los datos. Los autores declaran que han seguido los protocolos de su centro de trabajo sobre la publicación de datos de pacientes.

Derecho a la privacidad y consentimiento informado. Los autores han obtenido el consentimiento informado de los pacientes y/o sujetos referidos en el artículo. Este documento obra en poder del autor de correspondencia.

\section{Conflicto de intereses}

Los autores declaran que no existe conflicto de interés, puesto que no hay ninguna relación financiera 0 personal al momento de escribir o remitir el artículo con personas 0 instituciones que pudieran dar lugar a conflictos de interés, en relación con el artículo que se remite para su publicación.

\section{Bibliografía}

1. World Health Organization. Blindness and Visual Impairment. Actualizada 2006. Disponible en: http://www.who.int/features/factfiles/vision/04 en.html

2. Rengaraj V, David C, Radhakrishnan M, Kenia H, Pariskshit G,Sabyasachi S. Manual Small Incision Cataract Surgery: A Review. Asia Pacific Academy of Ophthalmology 2012;1: 113Y119.

3. Barojas W, SanJuan E, Ortega G. Reporte de una campaña quirúrgica de catarata Instituto Nacional de Salud. Rev Mex de Oftalmol. 2010;84(2):91-5.

4. Parikshit G, Madan D, Praveen K, Nirmalm D. Why Do Phacoemulsification? Manual Small-Incision Cataract Surgery Is Almost as Effective, but Less Expensive. Ophthalmology. 2007;114:965-68.

5. Amar A, Dhivya A. Cost-effectiveness of cataract surgery. Curr Opin Ophthalmol. 2011,22:15-8.
6. Parikshit G, Prachi A, Sucheta K, Rahul D, Shilpa J, Madan D. Comparison of endothelial cell loss after cataract surgery: Phacoemulsification versus manual small-incision cataract surgery. Six-week results of a randomized control trial. J Cataract Refract Surg. 2010;36:247-53.

7. Rohit A, Srivalli K, Ghanshyam P, Virender S. Comparative outcomes of manual small incision cataract surgery and phacoemulsification performed by ophthalmology trainees in a tertiary eye care hospital in India: a retrospective cohort design. BMJ Open. 2012;2:001035-001136.

8. Jia-yu Z, Yi-fan F, Jian-qiu C. Phacoemulsification versus manual smaII-incision cataract surgery for age-related cataract: meta-analysis of randomized controlled trial. Clin Exp Ophthalmol. 2013;41:379-86.

9. Parikshit G, Sucheta K, Krishnaiah S, Rahul D, Shilpa J, Anand P. Safety and Efficacy of Phacoemulsification Compared With Manual Small Incisión Cataract Surgery by a Randomized Controlled Clinical Trial. Ophthalmology. 2005;112:869-74.

10. Peter Peduzzi, John Cocato, Elizabeth Kemper, Theodore R. Holford and Alvan R. Feinstein. A Simulation Study of the Number of Events per Variable in Logistic Regression Analysis. J Clin Epidemiol. 1996; 49(12):1373-9

11. Frank E. Harrell, Kerry L. Lee, David B. Matchar, and Thomas A. Reichert. Regression Models for Prognostic Prediction: Advantages, Problems, and Suggested Solutions. Cancer Treat Rep. 1985;69(10):1071-7.

12. Ellwein $L$, Urato $C$. Use of eye care and associated charges among the Medicare population: 1991-1998. Arch Ophthalmol. 2001;85:822-9.

13. Priya T, Sarada D, Nihal T. Endothelial Cell Loss and Central Corneal Thickness in Patients With and Without Diabetes After Manual Small Incision Cataract Surgery. Cornea. 2011;30:424-8.

14. Sambuddha G, Indranil R, Pradyot N, Dipankar M, Lakshmi K, Subhalakshmi M. Prospective randomized comparative study of macular thickness following phacoemulsification and manual small incision cataract surgery. Acta Ophthalmologica. 2010;88:102-6.

15. Manea G, Stanca H. Specular microscopy and clinical correlations. Ophthalmology. 2011;55(3):22-9.

16. Tuft S, Coster D. The corneal endothelium. Eye. 1990;4(3):389-424.

17. Moschos M, Chatziralli I, Sergentanis T. Viscoat versus visthesia during phacoemulsification cataract surgery: corneal and foveal changes. BMC Ophthalmol. 2011;11:4-7.

18. Rosado N, Natalie A. The changing fate of the corneal endothelium in cataract surgery. Curr Opin Ophthalmol. 2012;23:3-6.

19. Schultz R, Glasser D, Matsuda M. Response of the corneal endothelium to cataract surgery. Arch Ophthalmol.1986;104:1164-9.

20. Ventura A, Walti R, Bohnke M. Corneal thickness and endothelial density before and after cataract surgery. Br J Ophthalmol. 2001;85:18-20.

21. Fong $C$, Mitchell $P$, Rochtchina E, Cugati S, Hong T, Wang J. Three-year incidence and factors associated with posterior capsule opacification after cataract surgery: The Australian Prospective Cataract Surgery and Age-related Macular Degeneration Study. Am J Ophthalmol. 2014;157:171-9.

22. Ruit S, Tabin G, Chang D. A prospective randomized clinical trial of phacoemulsification vs manual sutureless small-incision extracapsular cataract surgery in Nepal. Am J Ophthalmol. 2007;143:32-8.

23. Seitzman G, Gottsch J, Stark W. Cataract surgery in patiens with Fuchs corneal dystrophy expanding recommendations for cataract surgery without simultaneous keratoplasty. Ophthalmology. 2005;112:441-6. 